\title{
One Hidden Object, Two Spatial Codes: Young Children's Use of Relational and Vector Coding
}

\author{
David H. Uttal and Lisa B. Sandstrom \\ Department of Psychology \\ Northwestern University \\ Nora S. Newcombe \\ Department of Psychology \\ Temple University
}

\begin{abstract}
An important characteristic of mature spatial cognition is the ability to encode spatial locations in terms of relations among landmarks as well as in terms of vectors that include distance and direction. In this study, we examined children's use of the relation middle to code the location of a hidden toy, using a procedure adapted from prior work on spatial cognition in gerbils (Collett, Cartwright, \& Smith, 1986). Children of 4 and 5 years searched for a hidden toy in a large-scale environment. They were trained to find the toy with either 2 or 1 landmark present. On subsequent trials we altered the number and locations of the landmarks to determine how children represented the location of the toy. With 2 landmarks present during the initial training trial, the children coded both the middle location and the distance and direction from the toy to the landmarks. With 1 landmark present during the training trial, the children coded the location in terms of distance and direction to the single landmark. Our results shed light on seemingly inconsistent prior findings in both human and nonhuman species and indicate that both relational and vector coding are present in young children.
\end{abstract}

Organisms keep track of spatial locations in many ways, based on characteristics as disparate as the polarizing properties of moonlight (Dacke, Nilsson, Scholtz, Byrne, \& Warrant, 2003) and deviations in the earth's magnetic field (Lohmann,

Correspondence should be sent to David Uttal, Department of Psychology, Northwestern University, 2029 Sheridan Road, Evanston, IL 60208-2710. E-mail: duttal@ northwestern.edu. 
Cain, Dodge, \& Lohmann, 2001). Individual organisms often encode locations in more than one way, and this redundancy may provide evolutionary advantages (Gallistel, 1990; Newcombe \& Huttenlocher, 2000; Roberts, 2001; Sherry \& Schacter, 1987). For example, honeybees typically use the position of the sun to locate food, but on cloudy days they use a backup system that relies on the positions of landmarks observed on previous trips (Dyer \& Gould, 1981). A substantial amount of research has been devoted to studying the ways in which organisms keep track of (or code) spatial locations.

We report here the results of an investigation of children's use of two ways of coding the locations of hidden objects. Both involve remembering the location of a target location in terms of the position of landmarks, but the way that the information is coded differs fundamentally. In vector coding, the target location is coded in terms of distance and direction from a target landmark. An example is shown in the left panel of Figure 1. Using the tree as a landmark, the organism would encode the direction to the tree and how far the object is from it. When two or more landmarks are present, the possibility of a distinctly different form of coding emerges. In relational coding, the organism locates an object on the basis of some aspect of the relation that is formed by two or more landmarks considered simultaneously. For example, the organism could code the location of the toy as "in the middle" of the configuration of the four trees shown in the middle panel of Figure 1. A middle relation is not tied to any specific distance or angle but is defined instead in terms of the relation between two or more landmarks (see Kamil \& Jones, 1997, 2000; MacDonald, Spetch, Kelly, \& Cheng, 2004). Thus, as shown in the right panel of Figure 1, this relation remains the same even when the distance between the landmarks is increased or reduced.

There is extensive research on the use of vector coding in both human and nonhuman species. Many nonhuman species use vector coding, and children can do so by the end of the second year (Bushnell, McKenzie, Lawrence, \& Connell, 1995; Newcombe, Huttenlocher, Drummey \& Wiley, 1998). There is, however, substantial debate regarding whether various species use relational coding and when this ability emerges in humans (Biegler, McGregor, \& Healy, 1999; Collett et al., 1986; Kamil \& Jones, 2000; MacDonald et al., 2004). For example, Collett et al. (1986) found that gerbils did not rely on the middle relation between two landmarks. The gerbils were first trained to search for a seed hidden between two landmarks (see Figure 2). The distance between the landmarks was then doubled. The gerbils did not search at the midpoint between the two landmarks. Rather than searching at a middle location, they searched at the locations that represented the distance between the seed and the landmark in the original training trials (see Figure 2). Collett et al. concluded that the gerbils did not use the relation between the two landmarks to find the hidden seed; instead, they coded the vector from the starting position to only one of the two landmarks.

Other species, however, do seem capable of relational coding. For example, Kamil and Jones $(1997,2000)$ trained Clark's nutcrackers (a member of the crow 


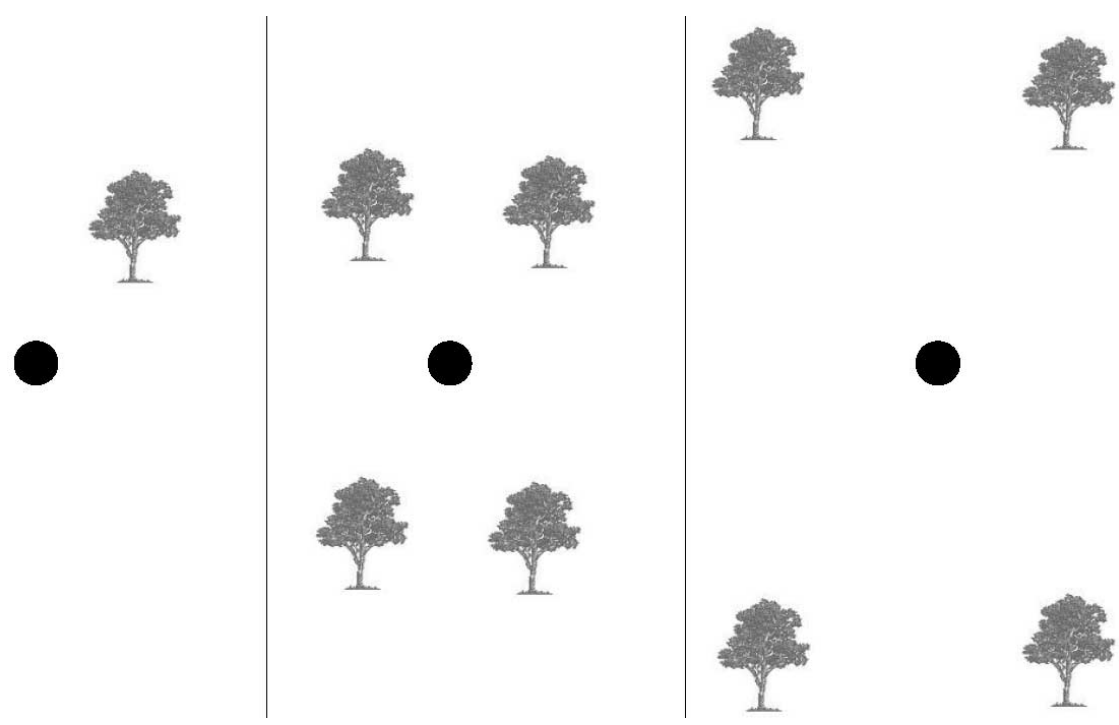

FIGURE 1 An example of vector coding and relational coding. The circle represents a hidden toy, and the trees represent landmarks. The left panel represents vector coding; the hidden object's location is coded in terms of distance and direction from the single tree. The middle panel represents relational coding; the hidden object is located in relation to the configuration formed by the four landmarks. The object is at the centroid of this configuration. The right panel represents a procedure for distinguishing distance and landmark coding. Expanding the positions of the landmarks alters the distance of the target from each of the landmarks, but the similarity relations among the landmarks are preserved. If the organism can find the toy after this transformation, then it must have coded the locations in terms of the relations among the landmarks.

family) to search at the midpoint between two landmarks by lengthening or shortening the distances between them. The training set included distances ranging from $38 \mathrm{~cm}$ to $98 \mathrm{~cm}$, in $20-\mathrm{cm}$ increments. Thus the birds learned to find the seeds with the landmarks separated by seven different distances. The birds were able to locate the hidden food, even when the landmarks were moved to distances that differed from those experienced during training. Kamil and Jones therefore concluded that Clark's nutcrackers, unlike gerbils, code a unique middle relation that is not tied to a particular vector from either landmark.

Our focus here is on the development of relational coding in children. We were interested in whether, and when, children can use the relation between two landmarks as the basis for locating a hidden toy. To our knowledge, no research has specifically investigated whether children can code a middle relation as did the Clark's nutcrackers in Kamil and Jones's $(1997,2000)$ research. However, prior research suggests that relational coding emerges no earlier than age 5, and possibly later (Gouteux \& Spelke, 2001; MacDonald et al., 2004). For example, MacDonald et al. found that, in some cases, children up to 9 years of age failed to use rela- 


\section{$x$}

FIGURE 2 Summary of procedures and results of Collett et al.'s (1986) work with gerbils. The top panel shows the location of the landmarks (represented by squares) and the hidden food (represented by an X) on the training trial. The bottom panel shows the locations of landmarks on the test trial and where gerbils searched on average (represented by circles) after the landmarks were moved.

tional coding to search for a hidden object at the centroid of four landmarks when the distance between landmarks was increased relative to the distance seen during training trials. The results of this study suggest that relational coding may emerge in children much later than vector coding does.

We suggest that prior research has assessed young children's relational coding using an unnecessarily stringent criterion. Researchers have consistently used a configuration of several landmarks to assess whether children can code relationally (Gouteux \& Spelke, 2001; MacDonald et al., 2004). Coding in terms of a configuration of several landmarks may be a sufficient but not necessary condition for demonstrating relational coding. As Kamil and Jones $(1997,2000)$ demonstrated with Clark's nutcrackers, a middle relation can be defined with only two landmarks. Although simpler than a coding that is based on a configuration of several landmarks, this form of middle is nevertheless inherently relational; the middle location between two landmarks stays the same even when the distance between them is altered. In this research, we tested whether young children could code the middle relation in a two-landmark search task. If obtained, this result would suggest that prior demonstrations of children's difficulty with relational coding were, in part, attributable to using information about a configuration of several landmarks, rather than to a general inability to think about and use any relational information.

Partial support for the claim that children could code simpler relational information can be found in prior research. For example, Quinn and colleagues (e.g., Quinn, Adams, Kennedy, Shettler, \& Wasnik, 2003; Quinn, Norris, Pasko, Schmader, \& Mash, 1999) found that infants as young as 6 months old can 
discriminate the relation between from the relations below and above. Infants were habituated to the position of a figure (e.g., a plus sign) below or above (or to the left or right of) two parallel lines. The researchers then moved the figure to a location between the lines. Infants dishabituated to the change, indicating that they could distinguish between from below and above. This ability was limited, however, to trials that involved the same figure. When the experimenters introduced a new figure (e.g., a plus sign was changed to a triangle), only 9- and 10-month-olds dishabituated. These results establish clearly that even infants can perceptually discriminate a between relation from other types of relations, which is a necessary precursor of relational coding. However, that infants can discriminate between from above or below does not necessarily mean that they can use the information to locate a hidden object. Quinn's results, therefore, are consistent with the possibility of relational coding, but children did not need to remember the specific location of an object, and hence we do not know if the infants coded its spatial location.

Additional research is also consistent with the claim that young children might be capable of relational coding. For example, Huttenlocher, Duffy, and Levine (2002) have argued that very young children's codings of extent are based on the relative amount of defined space occupied by an entity, such as a liquid or a cylinder in a container, rather than on absolute coding of height or length. Even young infants can distinguish and compare quantities, but they do so on the basis of relative proportion, rather than measuring the precise quantity or amount (Huttenlocher et al., 2002; Mix, Huttenlocher, \& Levine, 2003). These findings suggest that one might expect children to code spatial locations relationally at a young age.

Thus, there are reasons to hypothesize that children might use relational information to determine location by an early age, and yet the evidence on this point is far from clear. In this research we sought to provide more conclusive evidence regarding children's coding and use of a middle relation. A second goal was to determine whether children could simultaneously take into account both the relation between landmarks and the absolute distance between a hidden object and a single landmark.

To investigate these issues, we adapted the methods that have been used to investigate the coding of middle in nonhuman species. Our method was closely related to that of Collett et al. (1986). In fact, we scaled the testing area so that, relative to body size, there would be a rough comparability between our situation and the one that Collett et al. used with gerbils. Like Collett and colleagues, we trained our participants to find the hidden toy initially with either one or two landmarks present. When two landmarks are present, there are two ways to code the location of the toy: either in terms of the middle relation between the two landmarks or in terms of distance and angle from one of the two landmarks. To determine which form of coding children used, we then doubled the distance between the two landmarks. This change provided a strong test of whether children coded relationally or in terms of a single landmark. If children can code the middle relation, then they 
should continue to find the toy after the landmarks are displaced in the manner shown in Figure 2, because the middle location remains the same after the landmarks are displaced. If, however, children exclusively rely on vector coding, then they should search at either or both of the fixed distances from the two landmarks, and hence they should fail to find the toy. Following the two-landmark trial, we conducted additional trials to determine whether children also could find the toy when only one or no landmarks were present. When children are trained with only one landmark present, then they must use vector coding. Their behavior in this condition, where there is no possibility of relational coding, provides the necessary comparison and control for the two-landmark training condition.

\section{METHOD}

\section{Participants}

The participants were sixteen 4-year-olds $(M=53.7$ months; range $=48-58$ months) and sixteen 5-year-olds ( $M=65.1$ months; range $=60-72$ months). Each age group was composed of 8 boys and 8 girls. The children were recruited by direct mail to their parents. They lived in the western suburbs of Chicago; most were White and middle-class.

\section{Materials}

The experiment took place during the summer in an outdoor prairie preserve, with approximately $177 \mathrm{~m} \times 610 \mathrm{~m}$ of open land. The grass was not cut and grew to a height of approximately $50 \mathrm{~cm}$. There were some distant features that could potentially serve as landmarks, such as a small hill and telephone poles, but these were quite far away (almost $2 \mathrm{~km}$ in some cases). Given prior demonstrations that children less than 8 years do not accurately use distant landmarks to code location (Overman, Pate, Moore, \& Peuster, 1996), it seemed unlikely that the presence of such faraway landmarks would influence children's performance. Moreover, the experimental design included a control for this possibility (see the following section).

Two identical folding chairs served as landmarks. The hidden toy was a small plastic alligator, nicknamed "Ally," approximately $14 \mathrm{~cm}$ long and $6 \mathrm{~cm}$ wide. Golf tees were used to mark where the child searched. We measured distances with a metric measuring tape and recorded times with a stopwatch.

\section{Procedure}

The experiment began with a training trial, which was designed to help children understand the task and to establish an initial coding. We then conducted four test trials to determine how the children had represented the location of the toy. 
Training trial. The training trial was a critically important aspect of the experiment. On this trial, the children learned where the toy was relative to the position of one or two landmarks. We pointed out the landmarks to them and gave them multiple opportunities to encode the location of the toy relative to landmarks. Our goal was to create a mental representation of the toy's location that was based on the initial positions of the landmarks, and children were told that Ally would always be hiding in the same place throughout the game. Altering the positions of the landmarks on subsequent test trials, after the child had established the location of the toy, provided a way of indexing how the children represented this location.

Our primary focus is on whether young children can use the relation between two landmarks as a basis for spatial coding. To address this question, we had one group of children learn the location with two landmarks present. To provide a baseline and to assess whether the children could also use vector coding, we also asked a second group of children to learn the locations with a single landmark present. We refer to these conditions as the one-landmark and two-landmark training conditions.

Figure 3 shows the initial location of the landmarks for the two training conditions. In both conditions, the positions of the toy and of the starting location remained constant throughout the experiment; the positions of the landmarks were altered on different trials (see Figure 4). In the two-landmark training condition, the landmarks were placed $6 \mathrm{~m}$ apart along a row that was located $3 \mathrm{~m}$ from the toy. In the one-landmark training condition, the single landmark was placed at one of the two positions. Half of the children learned with the single landmark placed in the left position, and the other half learned with the single landmark placed in the right position. The starting location was $15 \mathrm{~m}$ from the toy (see Figure 3).

\section{One-Landmark Training Condition}

Toy

\section{Two-Landmark Training Condition}

घ

E

Toy

\section{Starting Location}

FIGURE 3 A schematic drawing of the locations of the starting position, the hidden toy, and the landmarks for the two conditions. 

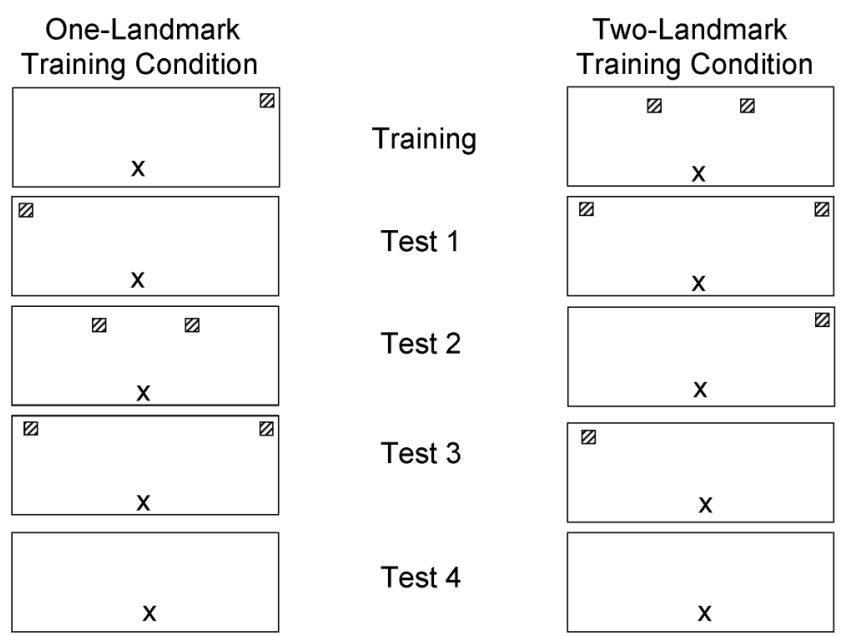

FIGURE 4 The positions of the landmarks (represented by squares) and the hidden toy (represented by an $\mathrm{X}$ ) on each trial in the two conditions. Note that the toy was always hidden in the same location on every trial in both conditions.

Aside from the number and positions of the landmarks, the procedures for the training trial were identical for the two conditions. The experimenter and child stood at the starting location. The experimenter showed the child the toy alligator and said, "Ally wants to play hide and seek in this field. Would you like to play hide and seek with Ally? I am going to hide her in the grass, and I need you to try to find her. Do you want to play this game?"

If the child agreed, the experimenter walked in a roundabout way to the hiding location. The child watched from the starting point as the experimenter hid the toy deep in the grass. It was not visible even up close, and the children had to move the grass to see the toy. While the experimenter stood near the hiding location, she said:

Now, look all around and pay close attention to what you see because you are going to have to find your way back to this spot to find Ally. See that chair over there? [If the child was assigned to the two-landmark training condition, the experimenter also pointed out the second chair]. Now look all around again and see where you are, because I will ask you to come back here to find Ally. When you think you know where Ally is hiding, I need you to put your hand on the ground and yell out, "I found her!" Now you are ready to play the game! When I say "Go," I want you to try to find Ally as fast as you can.

The experimenter then walked in a roundabout way back to the starting location where the child was waiting. The experimenter then asked the child to find the toy. 
A second experimenter placed a golf tee deep in the grass to indicate the first search that the child made, that is, the location at which the child first touched the ground with his or her hands. If the first search was incorrect, the child was asked to continue searching. If the child had not found the toy after $2 \mathrm{~min}$, the experimenter walked the child to the correct location. The entire procedure was then repeated until the child could find the toy without error within the 2-min time limit. Thus, all children eventually found the toy on the training trial.

Test trials. After the child had found the toy on the training trial, the experimenter put it back at the original location while the child watched. Then the experimenter and child began walking back to the starting location, with their backs turned to the chairs. Because the child could not see the landmarks, a second experimenter was able to surreptitiously change their positions, as shown in Figure 4.

On each test trial, before the number or positions of the landmarks were altered, the experimenter hid the toy in the same location and reminded the child that Ally would be hiding in the same place as before. The number or position of the landmarks was altered on each test trial to allow us to test how children coded the locations during the test trials and how they responded to changes in the available landmarks.

The changes differed for the two conditions. Because our primary focus is on whether children can use the relation between two landmarks to code a position, we first describe the locations of the landmarks in the two-landmark training condition, followed by those for the one-landmark training condition. Finally, we present the procedures that were used to record and score the searches.

Two-landmark training condition. For the first test trial in the twolandmark training condition, the distance between the landmarks was twice $(12 \mathrm{~m})$ as great as it had been during the training trial $(6 \mathrm{~m})$. This manipulation allowed us to answer our primary question: whether young children can use the middle relation between two landmarks to find a hidden object, independent of the distance between the landmarks. If children coded the location of the toy on the training trial as at the midpoint between the two landmarks, then they should perform well even when the distance between the landmarks is altered. However, if children only code the relation between the toy and one landmark, then they should search for the toy at the distance from a landmark at which it was hidden during the training trial when the landmarks were closer together (see Figures 1 and 2).

After the two-landmark trial, we tested children's performance with only one landmark and then with no landmarks (see Figure 4). These manipulations provided information about whether children also coded the original distance of the hidden toy to the landmark during the training trial. If children only coded relationally, then they might not code the distance from the landmark and hence would find it difficult to find the toy when a landmark was removed. On the final 
trial, we removed the landmarks. This allowed us to check our assumption that children could not find the toy on the basis of more distant landmarks (such as trees or hills). In addition, observing children's searches with no landmarks present allowed us to determine how close children could get to the toy solely on the basis of an egocentric, dead-reckoning strategy, which involves keeping track of time and rate of travel to estimate distance (Gallistel, 1990).

One-landmark training condition. Half the children in this condition were trained with the single landmark on the right, and the other half were trained with the single landmark on the left. The landmarks were placed in the same locations as in the two-landmark trial, but children in this condition only saw one of the two landmarks on the training trials. Figure 4 shows the positions of the landmarks for the children who were trained with the single landmark on the right. The single landmark was moved to the alternate right or left position on the first test trial.

In the remaining trials, we tested children's performance with two landmarks present and then with no landmarks. Introducing a second landmark allowed us to determine whether children would use the relation between two landmarks when they had not initially been trained on that relation.

Scoring the searches. We placed a golf tee in the grass at the location at which children first searched. If this location was incorrect, the child was allowed to continue to search for up to $2 \mathrm{~min}$, after which the experimenter showed the child the correct location. The location of children's searches were scored by imposing a Cartesian plane on the search space with the hidden toy serving as the reference location at $(0,0)$. We then calculated the distance between where the child first searched and the location of the hidden toy.

After each trial, the experimenter and child walked in a roundabout way back to the starting location. While the child's back was turned, the second experimenter set up the landmarks for the next trial.

\section{RESULTS}

We analyzed children's searches using two measures. The first measure was where they first searched, that is, the location at which they first touched the ground with their hands. Visual presentation of these initial searches highlights the patterns of performance. Inferential statistics were used to test specific hypotheses regarding the locations of these first searches.

The second measure was how many children found the toy within $2 \mathrm{~min}$. This is a more lenient criterion that allows for the possibility that children might initially search incorrectly but then go on to find the toy. In general, however, children tended either to find the toy on their first search or not at all. 


\section{Two-Landmark Training Condition}

Because our primary focus is on whether children can use the relation between the two landmarks as a basis for spatial coding, we present first the results of the two-landmark training condition. On Test Trial 1 (see Figures 4 and 5), we doubled the distance between the landmarks, from $6 \mathrm{~m}$ to $12 \mathrm{~m}$. There were no significant differences on any trial between the performance of 4- and 5-year-olds in terms of the accuracy or latency of searches, and hence these groups were combined in the graphs.

Moving the landmarks apart did not alter the middle location between the two landmarks, although it did radically alter the distance between each landmark and the hidden toy. Figure 5 indicates that children's searches were very accurate on this first test trial. Even though the distance between the landmarks was doubled, most children's first searches were very close to the target. The average distance from the locations at which children first searched and the correct location was only $0.18 \mathrm{~m}(S E=.08)$. Although small, this distance did differ significantly from a perfect search, that is, a search that is zero meters from the target, $t(15)=2.3, p<$ .05 . However, as Figure 5 reveals, this small difference was due only to a few children not searching at the precise location initially rather than to a failure to use relational coding. In addition, all children did go on to find the toy within the allotted $2 \mathrm{~min}$, with an average searching time of $49.9 \mathrm{sec}(S E=8.33)$. Thus, we conclude

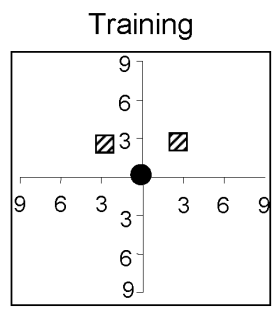

Test 1

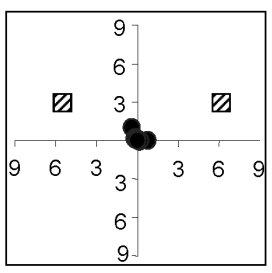

Test 2

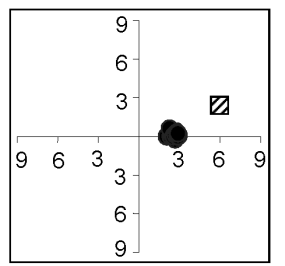

Test 3

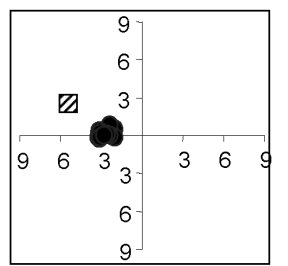

Test 4

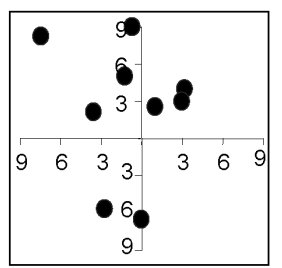

FIGURE 5 Children's first search attempts for the two-landmark training condition. The circles represent children's first searches, and the squares represent the locations of the landmarks. The data for two children are not shown on Test Trial 4 because these children searched more than $9 \mathrm{~m}$ from the target. 
that in contrast to gerbils, 4- and 5-year-olds can use the middle relation between two landmarks to find a toy.

We next examined performance on Test Trials 2 and 3, on which we removed one of the two landmarks (see Figures 4 and 5). On these trials, children's first searches were at precisely the locations that would be expected if they relied on distance coding (see Figure 5). These locations were $3 \mathrm{~m}$ to the right and left of the landmarks. Thus, simply removing one of the two landmarks led to a dramatic change in children's searches. In contrast to the two-landmark test trial (Test Trial 1), on which children relied on relational coding, their searches now reflected the original vector that they observed on the training trial. With one landmark in either the far right or in the far left position, children's first searches did not differ significantly $(M=2.9 \mathrm{~m}, S E=.07)$ from the expected distance from the target, $3 \mathrm{~m}, t(31)$ $=-1.4, p=.17, n s$. Because children were searching on the basis of the original vector that they had learned during the training trial, they did not find the toy because the landmarks were considerably farther from the toy than they had been on the training trial. In fact, only one child found the toy where it was actually hidden within 2 min.

Finally, we examined performance on Test Trial 4, on which we removed both landmarks. As shown in Figure 5, the children's searches did not form a consistent pattern, $(M=7.9 \mathrm{~m}, S E=1.1$; range $=2.5-13.2)$. Only $19 \%$ of the children found the toy within $2 \mathrm{~min}$. Children's poor performance on this trial confirms that they did not use distant landmarks, such as distant trees or telephone poles, to find the toy. If children were relying on these distal landmarks, then they could have performed well even when the more local landmarks (the chairs) were moved.

\section{One-Landmark Training Condition}

We turn now to the performance of children who were trained with only one landmark present. Recall that half the children in this condition were trained with the single landmark on the right, and the remaining children were trained with the landmark on the left. On Test Trial 1, we moved the single landmark to the alternate right or left position (see Figures 4 and 6). In the remaining trials, we tested children's performance with two landmarks present and then with no landmarks (see Figures 4 and 7). Introducing a second landmark allowed us to determine whether children would use the relation between two landmarks when they had not initially been trained on that relation.

As expected, on Test Trial 1, the children searched at the same distance and direction from the landmark as they had during the one-landmark training trial. When the single landmark was moved to the alternate left or right position, the location of children's first searches $(M=12.1 \mathrm{~m}, S E=.1)$ did not differ significantly from what would be expected if children relied on vector coding $(M=12 \mathrm{~m}), t(15)$ $=1.2, p=.3, n s$. As shown in Figure 6 , these locations were $6 \mathrm{~m}$ to the right and left 
Training, One Landmark, Right

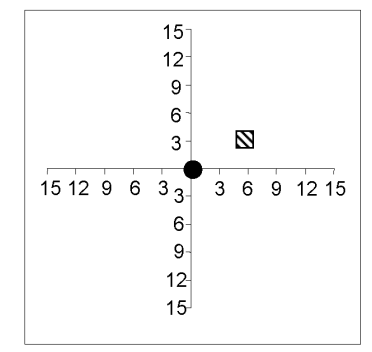

Test 1

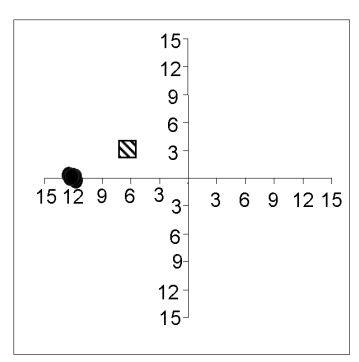

Training, One Landmark, Left

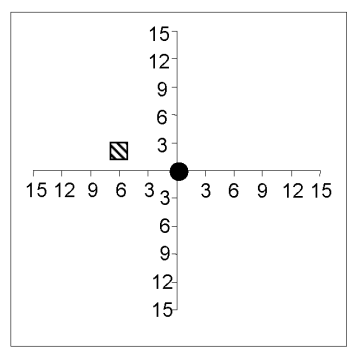

Test 1

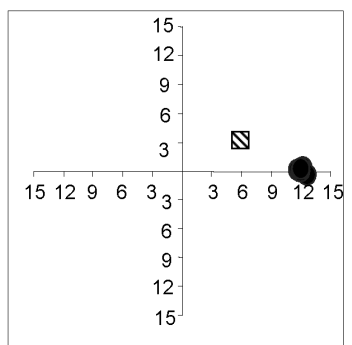

FIGURE 6 Children's first search attempts on the first test trial in the one-landmark training condition. The left panel shows the training configuration and results for children trained with the single landmark to the right. The right panel shows the same information for children trained with the single landmark to the left.

of the landmarks, placing them at $12 \mathrm{~m}$ from the correct target location. Only 3 of the 16 children found the toy within the 2-min limit, and these children succeeded by moving their hands through the grass in increasingly wider arcs.

\section{Two-Landmark Trials}

The two-landmark trials (Test Trials 2 and 3 ) in the one-landmark training condition differ fundamentally from those in the two-landmark training condition. In the one-landmark training condition, children had never seen two landmarks before this trial. Consequently, Test Trial 2 was the first time that relational coding could be used as a basis for locating the toy (see Figure 4). Thus it is not surprising that on the initial two-landmark trial, children performed poorly (see Test Trial 2 in Figure 7). The children searched with no distinct error pattern $(n=11 ; M=7.0 \mathrm{~m}, S E=.8$; range $=3.4-12.1)$ and none of the children found the toy within the 2-min limit. However, Figure 7 also indicates that children performed much better on Test Trial 3 , when we doubled the distance between the two landmarks. The error in their searches did not differ significantly from $0(M=.04 \mathrm{~m}, S E=.03), t(15)=1.4, p=$ 

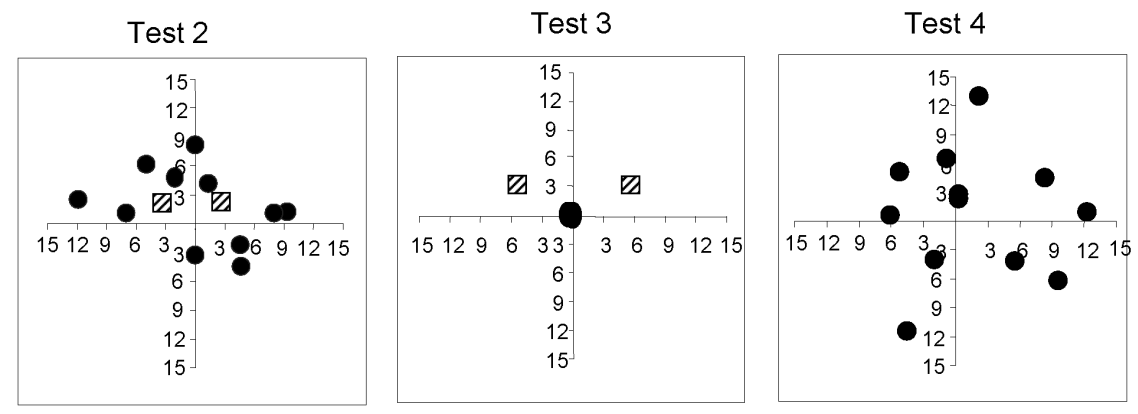

FIGURE 7 Children's first search attempts on Test Trials 2, 3, and 4 in the one-landmark training condition.

.19. Seventy-five percent of children located the toy on the first search, and the remaining $25 \%$ located the toy within $2 \mathrm{~min}$. The average search time was $43.0 \mathrm{sec}$ $(S D=27.3)$. This result is particularly noteworthy because it highlights that children must have relied on the relation between the two landmarks on the second two-landmark trial, but not on the first. Note that the children performed well only on the second two-landmark trial (Test Trial 3), even though the distance between the landmarks was substantially greater on the second two-landmark trial than on the first (Test Trial 2).

\section{No Landmarks}

As in the two-landmark training condition, children had difficulty finding the toy when we removed both landmarks (Test Trial 4) and did not demonstrate a consistent error pattern. Only $25 \%$ of the children found the toy within $2 \mathrm{~min}$. As shown in Figure 7, not one of the children found the toy on the first search $(n=12 ; M=7.9$ $\mathrm{m}, S E=1.1$; range $=2.5-13.2$ ). Children in the two conditions performed very similarly on this trial; an independent two-sample $t$ test revealed no difference between children in the two experiments on this trial, $t(22)=-0.6, p>.05$, ns.

\section{DISCUSSION}

This research focused on the development of children's use of two spatial codes: vector coding and relational coding. Three important findings emerged. First, the results provide strong evidence that 4-year-olds can use the relation between two landmarks as the basis for finding a hidden toy in a large, open space. Second, the results confirm that children also can code the distance and angle (i.e., the vector) from a single landmark, again in a large, open space. Third, the results also suggest 
that children of this age are capable of coding simultaneously on the basis of both the relation between pairs of landmarks and the vector of the hidden toy to single landmarks. In this section, we first discuss the evidence that supports each of these claims (and that helps to rule out alternative possibilities). We then discuss the implications of the results for understanding how children use relational information to code spatial location and for resolving some apparent paradoxes in the literature.

\section{Coding in Terms of Two Landmarks}

We have shown that 4-year-olds can find a toy solely on the basis of the middle relation between two landmarks in a large, unbounded space. Unlike the gerbils in Collett et al.'s (1986) work, and like the nutcrackers in Kamil and Jones (1997, 2000) work, children in our experiment did use the relation between the landmarks to find the hidden toy. In addition, our results demonstrate that children, unlike nonhuman species, can use the relation between two landmarks with only minimal training. Support for this claim comes from children's performance on the first test trial in the two-landmark training condition. Initially, we trained children with two landmarks close together. Then, immediately before the first test trial, we doubled the distance between the landmarks. This manipulation altered the distance between the landmarks but not the midpoint relation. Nevertheless, children searched very close to the correct location. This result suggests that children coded the middle location, as this location stayed the same despite the movement of the landmarks.

The results are inconsistent with other possible codings of the location. For example, the results cannot be explained in terms of the presence of distal landmarks, such as telephone poles or hills. Had children been relying on distal landmarks, then they should have performed well on all trials, regardless of the placement of the local landmarks, the chairs. However, this was not the case; children's performance was influenced greatly by the availability and movement of the chairs. The results also indicate that children's performance could not be based solely on an egocentric, dead-reckoning coding that involved keeping track of how far one traveled straight ahead from the starting location to the toy and traveling approximately that far each time. If children relied solely on dead reckoning, then we would expect them simply to run to approximately the same location on each trial (see Klatzky, Loomis, Beall, Chance, \& Golledge, 1998). This did not occur; when there were no landmarks present, children failed to find the toy, and the movement of the landmarks reliably affected their searches. This does not mean that children did not consider or code the distance between the starting location and the general area of the toy; an egocentric, dead-reckoning strategy could have gotten children to the general area in which we hid the toy. However, this form of coding could not in itself account for children's performance. For these reasons, we conclude that 
the children must have taken into account the relation between the toy and the local landmarks.

Our explanation for results assumes that the children maintained their representations of the position of the hidden toy relative to the position of the landmarks on the training trial. The initial training trial established a particular mental representation, relative either to two landmarks or to one landmark. The movements of the landmarks on subsequent trials were designed to assess what information was included in the mental representation that the children acquired during the training trial. It is possible, however, that children could have updated their representation of location during the testing trials. Whenever they were shown the location of the toy following a test trial, they might have noted where it was relative to the current position of the landmarks, rather than to the original positions on the training trials.

We believe for two reasons that our results generally reflect the learning that occurred on the training trial. First, only on the training trial were children specifically encouraged to code the relation between the toy and the landmark (or landmarks). We pointed out the landmarks on the training trial and had the children learn to find the hidden toy. If necessary, they were given multiple training trials until they found it. In contrast, any learning on the testing trials would have been incidental. The children were not trained to find the toy relative to the new positions of the landmarks, and the children did not seem to look at the landmarks after they found, or were shown, the toy. Second, the bulk of the results indicate that the children's searches were based on what they learned on the training trial, not on subsequent testing trials. For example, consider the results of Learning Trial 2 in the two-landmark training condition (see Figures 4 and 5). The children's behavior on this trial reflected the vectors from the toy to the positions of the landmarks on the training trials and not the vectors on Test Trial 1. Put another way, the children searched incorrectly because their searches were based on what they learned on the training trial rather than on what they could have learned about the landmark positions on the test trial. Other searches are also consistent with this interpretation.

There is one exception to this general claim, concerning Test 3 in the one-landmark training condition. We suggest that this is the one trial in which children did use information that they gained during the test trials. Test Trials 2 and 3 in the one-landmark condition differed from all others because they provided a basis for children to activate the possibility of thinking about the middle relation, which had not been a possibility during training. We suggest that the similarity and differences in the 2 two-landmark trials in the one-landmark training condition (Test Trials 2 and 3; see Figure 4) may have highlighted the value of the relational coding for finding the toy. On the first two-landmark trial in this condition, the children had no basis for using the relation between two landmarks as a basis for coding because they had been trained with only one landmark present (see Figures 4 and 7). However, when they failed to find the toy, the children were shown the correct location. At this point, they may have initially noted that the toy was in the 
middle between the two landmarks. A critically important factor that may have made this possible is that the landmarks were relatively close together on this trial (see Figure 4). Thus, in contrast to other test trials, the first two-landmark trial in the one-landmark training condition gave the children an opportunity to see the landmarks close to the toy during a test trial. Then, when we moved the landmarks farther apart (for the second two-landmark trial), they noted the similarity and differences between the two sets of landmark locations. In other words, seeing two landmarks first relatively close together and then relatively far apart may have made the children attend to the possibility of a relational coding. This sort of alignable difference has been shown to help children think about relations, both in search tasks (Loewenstein \& Gentner, 2001) and in solving analogies (Kotovsky \& Gentner, 1996). On the second two-landmark trial, children were now primed to the possibility of using both landmarks, and hence they searched correctly.

A possible concern regarding our interpretation is that children might have been able to find the toy on the two-landmark trials in the one-landmark training condition solely on the basis of landmark coding. Note that on Test Trial 3, the children had seen one of the two landmarks in the same position during the training trial (see Figure 4). It could be argued that children could then use the vector that they learned originally to find the toy. However, using the vector in this way would not necessarily lead to searching in the middle between the two landmarks. If children thought only of a vector to one landmark, then they should be equally likely to search at the location that maintained that vector to the opposite landmark. The results of Test Trial 1 of the same condition clearly establish that children will search at both these locations (see Figure 6). Because all children searched at the middle, we conclude that by Test Trial 3 they had gained insight into the possibility of relational coding.

\section{Vector Coding}

The results also confirm prior findings that children of this age can code the absolute distance from the target. The clearest evidence to support this claim comes from the one-landmark training condition. Children were first trained with a single landmark present, and we then moved the landmark to a position that required that children travel quite far from the true location of the toy to search based on vector coding. Nevertheless, children demonstrated that they coded the original distance from the target, in some cases moving from one side of the array to the other to preserve the angle and distance relation to the landmark that they had learned during training. Because the landmarks had been moved, they did not find the toy. However, their errors revealed that they knew the distance and angle from the landmark to the toy. The searches of children in the one-landmark training condition also rule out the possibility that performance was based on an egocentric strategy that involved simply walking straight ahead until they reached the landmarks. Children 
who were trained with only one landmark searched at locations that were (a) far away from the midline or the area directly ahead of the starting location, but (b) specifically predicted by a model of performance in which children's searches were determined by the distance and angle from the location of the toy to the original locations of the landmarks.

\section{Coding Both Vectors and Relations}

Finally, the results also suggest that children can use both the relation between the landmarks and the vector between a single landmark and the hidden toy. The results reveal that children in the two-landmark training condition knew, and used, both types of information. Recall that children who initially learned the location of the toy with two landmarks present were asked to search with only one landmark present on Test Trials 2 and 3 . On these trials, the children searched in locations that differed significantly from the search location on the previous (two-landmark) trial, despite the fact that the toy was always hidden in the same location. Even though they had found the toy (on the basis of a relational code) on the prior two-landmark trial, the children now failed to find the toy because they could no longer rely on the middle location, because there was only a single landmark present. On every single-landmark trial, children searched precisely at a location that was the same distance and angle from the landmark as during the training trial. The results suggest that they were now relying on vector coding; they reverted to the information about the distance and angle from the landmark that they observed during the training trial. In summary, the results reveal that children coded both the relation between the landmarks and the distance and the angle between the landmark and the toy. They also indicate that children regard relational coding as primary. In the first test trial in the two-landmark training condition, in which they could have used either kind of information, their first hypothesis was to rely on relational coding.

\section{What Defines "The Middle"?}

The discussion thus far supports the claim that 4-year-olds, unlike gerbils, could use the relation between the two landmarks as a basis for spatial coding and search. This raises the question of the form of the coding: How did the children use the relation between the landmarks to find the toy? In this section, we consider how children may have used the positions of the two landmarks as a basis for finding the hidden toy.

One possibility is that the children did not use relational coding or think about the middle relation per se, but rather based their correct searches at the middle location on the basis of statistical or neural properties that bias them to search at the middle. For example, one might argue that the middle location is the best estimate of the central tendency of the distribution of possible hiding locations (Biegler et 
al., 1999; Huttenlocher, Hedges, \& Duncan, 1991), or that the middle location can be defined in terms of patterns of neural excitation and inhibition that converge to specify a location that occurs when two landmarks are present (Schutte, Spencer, \& Schöner, 2003).

However, several aspects of the results and the design suggest that children's success on the two-landmark trials cannot be attributed solely to these statistical, perceptual, or neural aspects of the task. Most telling in this regard is the performance of children in the one-landmark training condition. As noted previously, these children failed to find the toy when we first introduced two landmarks (see results for Test Trial 2 in Figure 7). However, they did find the toy on the next two-landmark trial (Test Trial 3), when we moved the landmarks farther apart. If children could rely solely on the statistical best guess or the pattern of excitation and inhibition that would point them to the middle, then we would expect them to do well even when they were not trained with two landmarks present. Note also that on the first two-landmark trial (Test Trial 2) in the one-landmark training condition, the landmarks were relatively close together $(6 \mathrm{~m})$ (see Figure 4 and 7). Children performed poorly on this trial, but performed well on the second two-landmark trial (Test Trial 3), when we moved the landmarks father apart (12 $\mathrm{m})$. If they were searching on the basis of a bias or statistical best guess, rather than on coding a specific location, then they probably would have performed better with the landmarks closer together than farther apart, because the closer and more salient the landmarks were, the easier it would have been to accurately estimate the central tendency of the distribution of possible hiding locations. Likewise, the results cannot be explained in terms of averaging vectors from the individual landmarks (see Biegler et al., 1999). In contrast to prior research with nonhuman species, some of the children in this research experienced only one training distance, and thus they could not perform correctly by averaging distances that they had experienced during training.

The results, therefore, suggest that the children either coded or inferred the middle as a specific location. One way to do this would be to note the relation between the two landmarks and code the middle location as roughly halfway between the two landmarks. The children then maintained this relation when we moved the landmarks. Other research (Huttenlocher, Newcombe, \& Vasilyeva, 1999) has indicated that children are capable of this sort of informal proportional reasoning, and that even 3-year-olds can use a similar process to scale distances learned on a map. Further work is needed to specify precisely how organisms use the relation between two or more landmarks to code location. Nevertheless, this study suggests that 4-year-olds can use the relation between two landmarks as a reliable guide to location.

Our results leave open the possibility that younger children could code a middle relation. Testing younger children was not possible in our space because of younger children's limited height and mobility and the size of the field and 
height of the grass. Some research suggests that children as young as 3 years of age can use spatial relations to discriminate among locations, but only with extensive prior training, such as the use of relational language (e.g., above, middle, below; Gentner \& Loewenstein, 2002; Gentner \& Ratterman, 1991; Loewenstein \& Gentner, 2001).

\section{Coding Geometry}

Finally, the results help to shed light on prior results and to resolve some discrepant findings regarding when children may be able to use alternate coding strategies. Specifically, our results relate to the general issue of whether, and how, children use the geometry of spatial configurations as a basis for spatial coding.

Research on this question has led to seemingly discrepant findings, and the results presented here may provide a new perspective on the relevant questions. Some research has demonstrated that children as young as 18 months can use the geometry of a space to code the location of a hidden toy (Hermer \& Spelke, 1994, 1996). For example, Hermer and Spelke showed that children would search for a hidden toy either at the correct or geometrically identical corner in a rectangular-shaped room. In fact, even fish can use the geometry of an aquarium as a basis of spatial coding (Sovrano, Bisazza, \& Vallortigara, 2002). However, subsequent research (Gouteux \& Spelke, 2001) showed that children and nonhuman species used the geometry of the space only when it was made explicitly clear by the presence of surrounding walls or barriers (see also Kelly \& Spetch, 2001; Tommasi, \& Vallortigara, 2000; Wang \& Spelke, 2000). Children in these studies could not infer geometry based on the position of hiding locations; they only used geometry when it was extremely salient, defined by rigid walls. Similarly, MacDonald et al. (2004) found that children less than age 7 could not reliably use the configuration of a set of objects to locate a hidden toy. Thus there appears to be a fundamental difference between using the geometry of the space when it is defined by rigid boundaries (e.g., the walls of a room or of an aquarium) and when the geometry must be inferred based on the spatial relations among a set of landmarks.

Why do very young children succeed in using the geometry of a space when it is defined by rigid boundaries but fail when they must infer the geometry among a set of locations? We suggest that children less than 5 years may have difficulty thinking simultaneously about individual locations and about the relation of these landmarks to a configuration as a whole. To infer a configuration, children may need to think simultaneously about the individual landmarks and the configuration that they form. They must go beyond the information provided to imagine a configuration that is not explicitly provided (Gopnik \& Rosati, 2001; Uttal, Gregg, Chamberlin, \& Sines, 2001; Zelazo, Carter, Reznick, \& Frye, 1997; see also Pani, Mervis, \& Robinson, 1999, for a similar discussion of the spatial deficit in Wil- 
liams syndrome). Consequently, the relations among the landmarks that could serve as the basis for relational coding may not be obvious to young children. This study demonstrates, however, that this does not mean that young children are incapable of relational coding. With only two landmarks present, the relation between them may be more salient and easier to use; children do not have to think about an implied configuration of several landmarks but instead only about the relation between two landmarks.

\section{CONCLUSION}

The finding that children coded both the midpoint between the two landmarks and the distances from the toy to the individual landmarks is consistent with theoretical perspectives that emphasize flexibility in children's coding of spatial locations (Newcombe \& Huttenlocher, 2000; Plumert, 1994). Children develop the ability to code locations in multiple ways at an early age. Therefore, development cannot be explained solely in terms of the acquisition of more sophisticated representations. Instead, development consists of increasing flexibility in using different forms of coding and in knowing when one form of coding may be better or more appropriate than another. In these studies, we demonstrate that by age 4, children can code locations both in terms of relations among landmarks and in terms of distance and angle from a sole landmark. We have also demonstrated that relational coding is primary - that children will rely on the relational information when both forms of coding could be used to find the toy. Our results indicate that relational coding is present by age 4 and that children can code a single location both in terms of distance from a single landmark and in terms of a middle relation.

\section{ACKNOWLEDGMENTS}

This research was funded by Grants NSF 0087516, NIH R29HD34929, and IES R305H020088.

Portions of this work appeared in a senior honors thesis submitted by Lisa Sandstrom to the Department of Psychology, Northwestern University.

We thank Dedre Gentner, Janellen Huttenlocher, Susan Levine, Tracy Solomon, and Alison Lewis for comments on the work. We also thank Meredith Amaya, Deborah Farr, Maeve Hennerty, Mary Huft, JoAnn Sandstrom, Les Sandstrom, Karin Sandstrom, Ruth Swanson, and the children and parents who participated. Finally, we thank the Park District of Wheaton, Illinois, for allowing us to use portions of the Danada Equestrian Center. 


\section{REFERENCES}

Biegler, R., McGregor, A., \& Healy, S. D. (1999). How do animals 'do' geometry? Animal Behavior, 57, F4-F8.

Bushnell, E. W., McKenzie, B. E., Lawrence, D. A., \& Connell, S. (1995). The spatial coding strategies of one-year-old infants in a locomotor search task. Child Development, 66, 937-958.

Collett, T. S., Cartwright, B. A., \& Smith, B. A. (1986). Landmark learning and visual-spatial memories in gerbils. Journal of Comparative Physiology A, 158, 835-851.

Dacke, M., Nilsson, D., Scholtz, C., Byrne, M., \& Warrant, E. (2003). Animal behavior: Insect orientation to polarized moonlight. Nature, 424, 33 .

Dyer, F. C., \& Gould, J. L. (1981). Honey bee orientation: A backup system for cloudy days. Science, 214, 1041-42.

Gallistel, C. R. (1990). The organization of learning. Cambridge, MA: MIT Press.

Gentner, D., \& Loewenstein, J. (2002). Relational language and relational thought. In E. Amsel \& J.P. Byrnes (Eds.). Language, literacy, and cognitive development: The development and consequences of symbolic communication (pp. 87-120). Mahwah, NJ: Lawrence Erlbaum Associates, Inc.

Gentner D., \& Ratterman, M. (1991). Language and the career of similarity. In S. Gelman \& J.P. Byrnes (Eds.), Perspectives on thought and language: Interrelations in development (pp. 225-277). New York: Cambridge University Press.

Gopnik, A., \& Rosati, A. (2001). Duck or rabbit? Reversing ambiguous figures and understanding ambiguous representations. Developmental Science, 4, 175-183.

Gouteux, S., \& Spelke, E. (2001). Children's use of geometry and landmarks to reorient in an open space. Cognition, 81, 119-148.

Hermer, L., \& Spelke, E. S. (1994). A geometric process for spatial reorientation in young children. Nature, 370, 57-59.

Hermer, L., \& Spelke, E. S. (1996). Modularity and development: The case of spatial reorientation. Cognition, 61, 195-232.

Huttenlocher, J., Duffy, S., \& Levine, S. (2002). Infants and toddlers discriminate amount: Are they measuring? Psychological Science, 13, 244-249.

Huttenlocher, J., Hedges, L. V., \& Duncan, S. (1991). Categories and particulars: Prototype effects in estimating spatial location. Psychological Review 98, 352-376.

Huttenlocher, J., Newcombe, N., \& Vasilyeva, M. (1999). Spatial scaling in young children. Psychological Science, 10, 393-398.

Kamil, A. C., \& Jones, J. E (1997). The seed-storing corvid Clark's nutcracker learns geometric relationships among landmarks. Nature, 390, 276-279.

Kamil, A. C., \& Jones, J. (2000). Geometric rule learning by Clark's nutcrackers (Nucifraga columbiana). Journal of Experimental Psychology: Animal Behavior Processes, 26, 439-453.

Kelly, D., \& Spetch, M. (2001). Pigeons encode relative geometry. Journal of Experimental Psychology: Animal Behavior Processes, 27, 417-422.

Klatzky, R. L., Loomis, J. M., Beall, A. C., Chance, S. S., \& Golledge, R. G. (1998). Spatial updating of self-position and orientation during real, imagined, and virtual locomotion. Psychological Science, 9, 293-298.

Kotovsky, L., \& Gentner, D. (1996). Comparison and categorization in the development of relational similarity. Child Development, 67, 2797-2822.

Loewenstein, J., \& Gentner, D. (2001). Spatial mapping in preschoolers: Close comparisons facilitate far mappings. Journal of Cognition \& Development, 2, 189-219.

Lohmann, K. J., Cain, S. D., Dodge, S. A., \& Lohmann, C. M. F. (2001). Regional magnetic fields as navigational markers for sea turtles. Science, 294, 364-366.

MacDonald, S. E., Spetch, M. L., Kelly, D. M., \& Cheng, K. (2004). Strategies in landmark use by children, adults, and marmoset monkeys. Learning and Motivation, 35, 322-347. 
Mix, K., Huttenlocher, J., \& Levine, S. (2003). Quantitative development in infancy and early childhood. Infant \& Child Development, 12, 110-112.

Newcombe, N., \& Huttenlocher, J. (2000). Making space: The development of spatial representation and reasoning. Cambridge, MA: MIT Press.

Newcombe, N., Huttenlocher, J., Drummey, A., \& Wiley, J. (1998). The development of spatial location coding: Place learning and dead reckoning in the second and third years. Cognitive Development, 13, 185-200.

Overman, W. H., Pate, B. J., Moore, K., \& Peuster, A. (1996). Ontogeny of place learning in children as measured in the radial arm maze, Morris search task, and open field task. Behavioral Neuroscience, 110, 1205-1228.

Pani, J. R., Mervis, C. B., \& Robinson, B. F. (1999). Global spatial organization by individuals with Williams syndrome. Psychological Science, 10, 453-458.

Plumert, J. M. (1994). Flexibility in children's use of spatial and categorical organizational strategies in recall. Developmental Psychology, 30, 738-747.

Quinn, P., Adams, A., Kennedy, E., Shettler, L., \& Wasnik, A. (2003). Development of an abstract category representation for the spatial relation between in 6- to 10-month-old infants. Developmental Psychology, 39, 151-163.

Quinn, P. C., Norris, C. M., Pasko, R. N., Schmader, T. M., \& Mash, C. (1999). Formation of a categorical representation for the spatial relation between by 6- to 7-month-old infants. Visual Cognition, 6 , 569-585.

Roberts, W. A. (2001). Spatial representations and the use of spatial codes in animals. In M. Gattis (Ed.), Spatial schemas and abstract thought (Vol. 6, pp. 16-44). New York: Norton.

Schutte, A. R., Spencer, J. P., \& Schöner, G. (2003). Testing the dynamic field theory: Working memory for locations becomes more spatially precise over development. Child Development, 74, 1393-1417.

Sherry, D., \& Schacter, D. (1987). The evolution of multiple memory systems. Psychological Review, 94, 439-454.

Sovrano, V. A., Bisazza, A., \& Vallortigara, G. (2002). Modularity and spatial reorientation in a simple mind: Encoding of geometric and nongeometric properties of a spatial environment by fish. Cognition, 85, B51-B59.

Tommasi, L., \& Vallortigara G. (2000). Searching for the center: Spatial cognition in the domestic chick (Gallus gallus). Journal of Experimental Psychology: Animal Behavior Processes, 26, 477-486.

Uttal, D. H., Gregg, V., Chamberlin, M., \& Sines, A. (2001). Connecting the dots: Children's use of a systematic figure to facilitate mapping and search. Developmental Psychology, 37, 338-350.

Wang, R. F., \& Spelke, E. S. (2000). Updating egocentric representations in human navigation. Cognition, 77, 215-250.

Zelazo, P., Carter, A., Reznick, J., \& Frye, D. (1997). Early development of executive function: A problem-solving framework. Review of General Psychology, 1, 198-226. 